\title{
Las nuevas redacciones, el 'Big Data' y los medios sociales como fuentes de noticias
}

\author{
Denis ReNó \\ Universidad Estadual Paulista - UNESP (Brasil) \\ denis.reno@faac.unesp.br \\ Luciana RENÓ \\ Universidad Complutense de Madrid - UCM (España) \\ luciana.lorenzi@gmail.com
}

Recibido: 16 de julio de 2015

Aceptado: 18 de septiembre de 2015

\begin{abstract}
Resumen
Desde el surgimiento de la Internet, el posterior desarrollo de la web 2.0 y sus posibilidades narrativas, el periodismo ha dejado de ser lo mismo gracias a los espacios compartidos (medios sociales) y a la investigación de contenidos por algoritmos (Big Data). El artículo propone, basándose en una investigación bibliográfica y documental, además de la observación empírica del mercado y desde el punto de vista del participante, una comprensión sobre la composición de las redacciones contemporáneas y la producción de contenidos transmedia al mismo tiempo que pretende dar una visión de datos a partir de los medios sociales. Se espera por lo tanto proponer una reflexión sobre la relación profesional y cognitiva entre periodistas y profesionales del campo de la tecnología.
\end{abstract}

Palabras clave: Comunicación, Periodismo, Tecnología, Medios sociales, Narrativa transmedia.

\section{The newsroom, Big Data and social media as information sources}

\begin{abstract}
Since the emergence of the Internet, the further development of Web 2.0 and its narrative possibilities, journalism is no longer the same thanks to shared spaces (social media) and research of contents algorithms (Big Data). The article proposes, based on a bibliographic and documentary research, and empirical observation of the market and from the point of view of the participant, an understanding of the composition of contemporary newsrooms and production of transmedia content while intended to give a view data from social media. It is expected therefore to propose a reflection on the professional and cognitive relationship between journalists and professionals in the field of technology.

Keywords: Communication, Journalism, Technology, Social media, Transmedia storytelling.

\section{Referencia normalizada}

RENÓ, Denis y RENÓ, Luciana (2015): “Las nuevas redacciones, el 'Big Data' y los medios sociales como fuentes de noticias". Estudios sobre el Mensaje Periodístico. Núm. especial diciembre, págs.: 131142. Madrid, Servicio de Publicaciones de la Universidad Complutense.
\end{abstract}

Sumario: 1. Introducción. 2. Metodología. 3. Las redacciones tradicionales. 4. El algoritmo como herramienta para la "big" investigación de datos. 5. Las redacciones contemporáneas. 6. Los medios sociales y la noticia. 7. Conclusiones. 8. Referencias bibliográficas.

\section{Introducción}

Los cambios que involucran el periodismo contemporáneo provocan una necesaria revisión de las diversas características de la profesión, desde los procesos de construcción de la opinión pública por parte del lector/usuario hasta la configuración de las redacciones y las funciones que en ellas se definen. El escenario contemporáneo ofrece una nueva visión sobre la formación básica del periodista y provoca cambios en la filosofía de la profesión, como la exclusividad del ejercicio de la actividad por parte de los periodistas graduados y la circulación de la información a partir de los medios. 
Estos cambios implican, básicamente, el surgimiento de tecnologías y narrativas que definen los nuevos formatos y procesos de representación de la noticia y la búsqueda de informaciones que se integran en las nuevas plataformas de comunicación y en los dispositivos contemporáneos, interactivos y móviles y exigen una relación entre el conocimiento del contenido y el dominio de la tecnología. Una de las variaciones más expresivas se encuentra en el proceso de recogida y cruce de datos informativos - una evolución del método RAC ${ }^{1}$ (Reportagem Assistida por Computador), denominado por Dader (1997) como Periodismo Asistido por Ordenador, resultante del desarrollo del fenómeno Big Data, es decir, datos propios y limpios, presentes en la Internet y que sirven como fuente de análisis, interpretación y reconstrucción de la información (Renó y Flores, 2014). Este método exige un conocimiento sobre tecnología de la información, específicamente sobre el algoritmo. Otro cambio sustancial se evidencia en el campo del lenguaje comunicacional contemporáneo, que envuelve los tradicionales recursos hipermedia. Con el desarrollo de las narrativas transmedia por parte de los ciudadanos (Renó, 2014), ese lenguaje ocupa un espacio cada vez más expresivo en los procesos mediáticos. Sin embargo, para el desarrollo de estos contenidos es necesaria la presencia de expertos en tecnología, especialmente para trabajar con la construcción de los nuevos espacios o definir nuevos procesos de investigación de datos por algoritmos.

De hecho, las corrientes conservadoras que estudian el periodismo consideran fundamental que esa actividad sea exclusiva para los periodistas graduados en la profesión. La misma opinión es defendida por periodistas profesionales que, tal vez en búsqueda de una reserva de mercado o por la defensa de los dogmas de la profesión, reciben de manera negativa a los profesionales resultantes de otras profesiones en las redacciones. Es una actitud incoherente con la realidad presentada en el párrafo anterior, inevitable en el mundo de la noticia contemporánea, donde los medios sociales son espacios de descubrimiento de diversas informaciones de interés público.

El presente artículo ofrece una discusión sobre las tendencias de la profesión, especialmente el Big Data como procedimiento y los medios sociales como fuentes, además de dar una visión sobre la necesidad de una ampliación de funciones dentro de las redacciones. Son cambios que están siendo puestos en práctica por periódicos de Estados Unidos e Inglaterra, y empiezan a ser visibles en otros países, como Brasil, al mismo tiempo que comienzan a ser percibidos en programas universitarios que buscan una actualización de sus proyectos pedagógicos y, en sintonía con el mercado, mezclan conocimientos periodísticos con teorías y técnicas de sistemas de información.

\section{Metodología}

Para el desarrollo de la investigación se emplearon como métodos científicos la investigación bibliográfica y documental, además de la observación empírica del mercado en sí. Por otro lado este artículo se ha basado también en la observación participante

1 Como debate Nilson Lage (2003), el método RAC es una práctica periodística que se aproxima a las técnicas y las teorías de la Ingeniería de la Computación, obligando a una aproximación entre periodistas y profesionales de ese campo profesional. 
para entender estas transformaciones, específicamente en lo que a la producción de contenidos transmedia y la recolección de datos a partir de medios sociales se refiere.

A partir de esto, se han estudiado ejemplos sobre la utilización de medios sociales en la investigación periodística, además de ejemplificar situaciones en las que se pone de manifiesto cómo las redacciones han adoptado la contratación de profesionales de tecnología para trabajar como periodistas. Se trata de una tendencia que, a partir de los ejemplos presentados, queda clara y apunta hacia el desarrollo futuro de la profesión, tanto en el campo de los procedimientos como en la estructura de las empresas de comunicación periodística.

Las expectativas, tras los resultados obtenidos del estudio, denotan que la relación profesional existente entre periodistas y profesionales de tecnología está aceptada por las corrientes que no creen en un periodismo interdisciplinario, un hecho que puede ser considerado positivo a la hora de ejercer el periodismo, puesto que, de esta manera, podrán aprovecharse mejor las técnicas de investigación, como podría ser el caso de los medios sociales - un reflejo social en un mundo virtual.

\section{Las redacciones tradicionales}

Tradicionalmente, las redacciones se han constituido por un equipo de periodistas que, a partir de conceptos apoyados en las ciencias sociales, desarrollaron sus actividades en búsqueda de informaciones y de la construcción de contenidos que representen de alguna manera u otra la realidad observada, o compilada. Esos equipos, compuestos por periodistas graduados y por profesionales prácticos (destacados en la profesión, aunque sin formación específica), siguen técnicas que se aproximan al origen del periodismo: la búsqueda de informaciones acudiendo directamente a las fuentes y/o a los documentos. A pesar de ello, desde el surgimiento de la Internet el contacto con la noticia quedó lejano. Nacieron así los llamados "periodistas de oficina", que no salen de sus redacciones en búsqueda de noticias porque consideran la Internet como un canal eficaz en la relación con las fuentes, teniendo como herramienta el teléfono o, recién implantado, el WhatsApp. Estos canales son relativamente confiables si tenemos en cuenta que los niveles de interactividad (Thompson, 1998) ofrecidos son limitados. No podemos observar las dejas simbólicas presentes en la fisionomía de quien habla. Tampoco podemos tener la certeza de que la persona responsable del diálogo desde el otro lado del teléfono (por ejemplo, en el caso del WhatsApp) es la misma con quien pensamos dialogar.

Sin embargo, con la popularización de datos abiertos en la Internet, el periodismo ha ganado un nuevo aliado en su práctica profesional: el periodismo de datos. Sandra Crucianelli, cuando administraba el taller de periodismo de datos de la Fundación Knight Center, declaró considerar que en realidad el periodismo de datos es lo mismo que el periodismo de base de datos, pero se le aplica una denominación más sencilla. También defiende que el periodismo de datos posee un poco de las características de otras modalidades de periodismo, como el periodismo de investigación (pues adopta técnicas propias de ello), el periodismo de profundidad, el periodismo de precisión y el reportaje asistido por ordenador. Es casi siempre necesario analizar los datos y utilizar plantillas de cálculos - el periodismo analítico - muy útil cuando se trabaja con 
métodos analíticos para llevar esos datos a los sistemas de información geográfica. Además de esa mezcla de "periodismos", también están involucrados en el periodismo de datos el gran volumen de información y la visualización interactiva. Por eso, el involucramiento de un profesional de tecnología se justifica al equipo de redacción para desarrollar tareas como extracción de datos, depuración, aplicaciones de noticias, entre otras actividades.

Los productos que pueden resultar del trabajo periodístico de datos son al menos de cuatro tipos: artículos basados en datos, visualizaciones de datos interactivos, conjunto de datos abiertos y aplicaciones de noticias (News Apps). Esta posibilidad cognitiva es defendida por Manovich (2013: 337) como epistemología del software, o sea, "cuando el metamedio informático es caracterizado por su extensibilidad permanente".

Sin embargo, a pesar de poseer una proximidad con la tecnología, pocas redacciones aceptan la existencia de profesionales especializados en tecnología de procesamiento de datos en sus espacios profesionales. Esa defensa por el espacio periodístico era coherente en un momento cuando las noticias eran representadas por lenguajes tradicionales y las técnicas de investigación y de cruce de datos se limitaban al contacto visual, características que dejaron de ser una máxima del periodismo hace prácticamente 20 años, debido al surgimiento de la Internet y la utilización de la misma como herramienta de contacto, fuente de información y repositorio de datos. Aún así, algunas redacciones insisten en defender la hegemonía de profesionales graduados en periodismo en el ejercicio de la actividad profesional.

La justificación más común de esa defensa del espacio se ve respaldada por el conocimiento específico del periodismo - las técnicas de investigación y la selección de datos de interés público, así como los principios éticos que regulan la profesión. Obviamente, la observación de datos es algo que debe quedar bajo cuidado de los profesionales que tengan conocimiento sobre ello. Pero, las técnicas tradicionales no presentan eficacia alguna con las posibilidades existentes actualmente. Los datos están en las nubes, en la red, circulando en la Internet (o no, cuando se trata de datos propios). Es preciso definir los parámetros y los filtros para la búsqueda de datos y, por su parte, el aprovechamiento de los mismos.

También es fundamental construir contenidos que interactúan directamente con la sociedad a través de un lenguaje esperado por la audiencia, y no mediante una construcción de contenidos con lenguajes seculares que, hoy día, no ofrecen una producción de contenidos multiplataforma, además de no crear un marco de existencia de profesionales con un dominio claro de las tecnologías de construcción de espacios digitales, especialmente dirigidos a dispositivos móviles.

A pesar de ese escenario mutante, las diversas corrientes de periodistas y académicos afrontan la profesión como una actividad exclusiva del periodista. Sin embargo, el periodismo siempre fue interdisciplinar en su hacer, en su construir y en su reproducir. Es una actividad en equipo, con diversidad tecnológica y multiplicidad de lenguajes. Por esa razón, no aceptar la diversidad profesional es una incoherencia difícilmente explicable. 


\section{El algoritmo como herramienta para la "big" investigación de datos}

Para comprender las nuevas maneras de hacer periodismo, es fundamental que el periodista conozca el significado de la palabra (y el procedimiento) "algoritmo". Suele ser un término que no forma parte de la realidad periodística, pero cuando se descubre cuál es su significado queda claro que es fundamental para el periodismo desde su existencia.

"Algoritmo" es aquello relacionado a toda y cualquier tarea esquematizada a ser realizada (como ocurre en el periodismo desde su surgimiento), pero, a pesar de ello, puede ser una noción acuñada especialmente a la búsqueda de informaciones en la red a partir de los conceptos de Big Data ${ }^{2}$. Son tareas destinadas a la búsqueda y el filtro de determinadas informaciones estrechamente relacionadas entre sí, una actividad para la cual los periodistas no tienen un conocimiento técnico, y, en estas condiciones otros profesionales les ganan espacio en las redacciones.

Algoritmo es una secuencia de pasos ordenados de manera lógica para la ejecución de una tarea finita o infinita. Básicamente, se trabaja con entrada(s), procesamiento y salida(s). Para que un computador pueda desempeñar una tarea es necesario que sea bien detallada, sin ambigüedad, en tiempo de ejecución establecido y con una condición final como objetivo. Los profesionales del campo de la tecnología poseen una habilidad natural para el desarrollo del raciocinio lógico, muy útil en el avance de un algoritmo, pero no se puede considerar una condicional para desarrollar este tipo de tarea.

A partir de un algoritmo bien elaborado, es posible que un profesional del campo de la tecnología ejecute su desarrollo en un lenguaje de programación - lenguaje que dialoga con el computador. Así, la tarea será ejecutada y el objetivo deseado será alcanzado. Cuanto más detallados son los parámetros establecidos y determinados dentro del algoritmo para que ese profesional lo convierta en programa, mejor será el resultado alcanzado.

En el caso del Big Data, como el volumen de datos es gigante - datos estructurados y no estructurados, variados y con una amplia velocidad - unidad trina del Big Data, el algoritmo es útil para auxiliar el procesamiento de toda esa cantidad y variedad de información. Es posible utilizar palabras clave, filtros, análisis, de acuerdo con el interés y la búsqueda hecha.

Debido a la cantidad, variedad y velocidad de las informaciones que están disponibles hoy, es necesario tener varios programas trabajando conjuntamente para llegar al objetivo planteado. Claro está, que el análisis final del resultado obtenido es fundamental para definir dónde se puede utilizar la información analizada. El estudio puede ser desarrollado conjuntamente entre el periodista y el profesional de tecnología, en una actividad interdisciplinaria.

2 Popularizado en 2013, el Big Data es un método de recolección, aprovechamiento y reconstrucción de informaciones disponibles en la nube de la Internet y que tiene como propuesta la utilización de datos sucios y limpios. 


\section{Las redacciones contemporáneas}

Estos nuevos modelos y herramientas para la producción de noticias, como el Big Data, han provocado cambios en la configuración de las redacciones de diversos países, a pesar del conservadorismo característico del periodismo en general. Esta nueva composición es el resultado de nuevos formatos narrativos y tecnologías involucradas en los procesos reconocidos, además de estar indirectamente relacionada con los nuevos modelos de negocio. De este modo, ejercer el periodismo y administrar los medios necesarios se complementan de alguna manera, dando lugar a una agilidad más expresiva en búsqueda de resultados.

Por esta misma razón, importantes medios de comunicación, como The New York Times, cuentan con Arthur Ochs Sulzberger Jr., graduado en Ciencias Políticas por la Universidad de Tufts, Massachusetts (E.U.A.), como editor o, en la posición de director de ejecutivo, con la colaboración de Dean Baquet, que ha estudiado periodismo en la Universidad de Columbia, pero ha abandonado el programa.

Siguiendo esta misma línea, diversas universidades estadounidenses buscan mezclar asignaturas sobre elaboración de algoritmos y/o programación de base de datos en los programas de formación de periodistas. La universidad de Columbia ha creado en 2014 una maestría en Comunicación que abarca diversas líneas de investigación y disciplinas sobre el tema, lo que refleja esa demanda y la necesidad de una mejor capacitación de los nuevos periodistas.

En su afán de observar el periodismo y sus transformaciones como práctica y negocio, el investigador peruano afincado en España, Jesús Flores (2014), presenta un perfil de los profesionales de redacción contemporánea. En su estudio, además de observar las redacciones y sus nuevas configuraciones, Flores cuestiona el comportamiento de los medios preocupándose por el futuro de los profesionales tradicionales. Según el autor:

"Diferentes perfiles profesionales son descritos y analizados por expertos y estudiosos en la evolución del periodismo, factor que demuestra como los medios se están adaptando a los nuevos entornos, de modo que si no lo hacen corren el riesgo de, sencillamente, desaparecer del espacio mediático. ¿Pero, como graduar periodistas multimedia para redacciones integradas?" (Flores, 2014: 89).

El autor defiende que "los periódicos dejan de ser empresas periodísticas para convertirse en organizaciones de noticias (de Newspapers a News Organizations) con nuevos desafíos, adoptando nuevas formas narrativas y desarrollando nuevas formas de hacer negocio" (Flores, 2014: 90). Esas nuevas narrativas, que transitan entre una hipermedia avanzada y una narrativa transmedia (Renó, 2014), exigen una aproximación conceptual entre el periodista y el profesional de tecnología de la información. Esa aproximación puede coexistir con la configuración de una redacción interdisciplinaria o a partir de la formación académica con syllabus actualizado, donde los contenidos de los dos campos (periodismo y tecnología de la información) se enseñen a los nuevos profesionales. Lo que queda claro es que el mercado debe prepararse para una reformulación de la actividad periodística, como puede ser observado en diversos medios. Según Alberto Cairo (apud Flores, 2014: 101), "algunas 
redacciones se reorganizan para un futuro en el que los datos se transformarán en un medio y en el que las técnicas narrativas tradicionales se fundirán con presentaciones interactivas complejas".

En realidad, esos cambios venían siendo prorrogados desde el surgimiento de la Internet. Tal vez por esa misma razón el periodismo es la actividad comunicacional que presenta el desarrollo más tardío, frente a otras actividades, como la publicidad, la ficción o la educación. Sin embargo, las universidades observan que hay una necesidad de mejora en esto sentido y empiezan a construir proyectos pedagógicos que apuntan hacia una interdisciplinariedad dirigida también a la tecnología, y no solamente a las ciencias sociales. Según Flores (2014: 106), esa preocupación ha llegado a diversas universidades, incluyendo las más tradicionales:

Ernest Sotomayor, asistente de servicios profesionales de la Escuela de grado de Periodismo de la Universidad de Columbia, menciona que todos los estudiantes de su programa de maestría profesional en Ciencia de la Escuela de Periodismo de la Universidad de Columbia se gradúan con destrezas multimedia básicas.

Aún así, según el autor, "para Eldra Gillman, directora de contrataciones y educación de la CBS Corp., cualquier estudiante con destrezas tecnológicas se encuentra muy arriba de quien necesita capacitación" (Flores, 2014: 106). Obviamente, este es el retrato del mercado que, unido con la universidad, está promoviendo una modificación en el perfil de las principales redacciones contemporáneas.

De hecho, algunas corrientes de mercadeo y académicas insisten en un conservadorismo sobre la configuración de las redacciones, sin tener en cuenta las nuevas prácticas y maneras de descubrir fuentes y noticias ni las tecnologías disponibles para construir los contenidos periodísticos contemporáneos. Según Luciano Martins Costa, periodista y columnista del portal Observatorio da Imprensa ${ }^{3}$, la noticia está más en las manos de las empresas de periodismo. Para Costa (2014: web) "el dominio de empresas de tecnología en la producción y distribución de contenido informativo y de opinión está creando una nueva esfera pública, cuyos controladores no están preocupados por la transparencia y la ética". Sin embargo, es importante cuestionar si las empresas de periodismo se responsabilizan de la transparencia y la ética, teniendo en cuenta algunos ejemplos recientes del periodismo brasileño (la entrevista con la presidenta Dilma Rousseff en las elecciones presidenciales de 2014 realizada por el noticiero Jornal Nacional, o la revista Veja, edición 2.397 de 24 de octubre de 2014, entre otros casos).

El miedo manifestado en el artículo de Costa se refiere a la decisión sobre lo qué es o no es noticia, así como la manera de destacarlo, todo a partir de conceptos de algoritmo. De hecho, tanto Costa como los autores a los que él mismo hace referencia en el artículo se equivocan al pensar que la propuesta de contratar profesionales de tecnología de la información en las redacciones está limitada a la creación de algoritmos capaces de escoger y destacar noticias, sin considerar que estos profesionales pueden actuar en conjunto con periodistas, puesto que están preparados para colaborar con la selección y el

3 Observatorio da Imprensa es un importante espacio mediático brasileño que debate sobre el periodismo en sí. Es una referencia sobre el tema en los medios de comunicación. 
análisis de lo que es o no es noticia. Se trata de un trabajo colectivo, donde cada uno hace lo que mejor sabe hacer, a partir de su preparación profesional. Esa colaboración laboral destaca también en otros campos profesionales, como la medicina. El periodismo, por su importancia social, saldría beneficiado de esa inevitable unión, especialmente en una sociedad que ofrece informaciones por diversos espacios, como puede ser a través de los medios sociales, y los comparte con sus grupos personales.

\section{Los medios sociales y la noticia: nuevo escenario}

Cuando pensamos en medios sociales y noticia, la primera cosa que nos viene a la mente es la circulación de la información. En realidad, los medios sociales constituyen una eficaz herramienta para promocionar la circulación de la información, pero no está limitada solamente a eso, si tenemos en cuenta el tema relacionado con el Big Data.

Podemos considerar que el Big Data es una estrategia de investigación de datos en la web a partir de algoritmos apropiados para el cruce de datos propios y limpios. La labor de cruzar los datos sigue un parámetro que contiene, además de los códigos de programación y la definición de tareas y secuencias, la definición de palabras claves o términos que son buscados. Eso ocurre, por ejemplo, al empezar una búsqueda en Google, pues lo que hacemos es definir las palabras y el programa contesta con los resultados sobre los temas en cuestión. La diferencia es que Google ofrece, normalmente, datos limpios y disponibles, oficiales o no, mientras el Big Data propone que se siga más allá de lo limpio.

En este sentido, Lev Manovich (2013) presenta la idea de que hoy en día el software sigue al mando, es decir, necesitamos del software para entender y desarrollar tareas en la web, no solamente para descubrir informaciones, sino también para realizar procedimientos personales o profesionales en la nube. El autor propone que:

Convertir todo en datos y usar algoritmos para analizarlo, cambia lo que significa conocer algo. Crea nuevas estrategias, unidas, conllevan a una epistemología del software. La epistemología es una rama de la filosofía que se interroga sobre lo qué es el conocimiento, cómo se adquiere y en qué medida un sujeto puede ser conocido. El código digital, la visualización de datos, los GIS, la búsqueda de información, las técnicas de aprendizaje automático, el constante incremento de la velocidad de procesadores y el descenso de sus costes, las tecnologías de análisis de big data, los medios sociales y otras partes del moderno universo tecno-social introducen nuevas formas de adquisición de conocimientos y, en el proceso, redefinen lo que es el conocimiento. (Manovich, 2013: 337-338)

Manovich propone el desarrollo de algoritmos para obtener resultados concretos a partir del concepto de Big Data. El trabajo de búsqueda en la nube se hace por los algoritmos desarrollados específicamente para la tarea.

Por ejemplo, siempre es posible inventar nuevos algoritmos (o nuevas formas de escalar algoritmos existentes para analizar más rápido el big data) que puedan analizar los datos existentes hoy en las formas en las cuales los algoritmos previos no podían. Como resultado, podemos extraer patrones adicionales y generar nuevas informaciones de los datos viejos ya analizados. (Manovich, 2013: 338) 
El autor también hace una discusión sobre la relación entre los usuarios y los medios sociales. Para Manovich, la utilización de estos espacios es casi libre y los usuarios publican lo que deciden publicar. Es un espacio donde las informaciones pueden surgir de manera natural. Según el autor:

Los desarrollos de los años 90 se han diseminado a cientos de millones de personas que están escribiendo blogs, subiendo fotos y videos a los sitios sociales, y usando de forma libre (o casi libre) herramientas de software de producción y de edición que hace algunos años costaban decenas de miles de dólares. (Manovich, 2013: 1)

De hecho, los medios sociales son responsables del descubrimiento de informaciones diversas por parte de los periodistas contemporáneos. Actuar como periodista sin tener una cuenta en los diversos medios sociales es como trabajar sin máquina para escribir en los años 1940. Las noticias fluyen en los medios sociales, y los ciudadanos tienen interés en hacerlas circular, como defiende Dan Gillmor (2005), para quien los ciudadanos contemporáneos son los "seres medio".

Adoptar estrategias de construcción de la noticia a partir de la recolección de informaciones desde los medios sociales es común en el periodismo internacional, especialmente en situaciones extremas. En el año 2012, los periodistas estadounidenses cubrieron la trayectoria (y la destrucción) del huracán Isaac a partir de informaciones que circulaban en los medios sociales, especialmente en Twitter y YouTube. A partir de eso, algunos periodistas pasaron a definir esa recolección de informaciones como periodismo híbrido, por la mezcla existente de actividades y procedimientos en la búsqueda de la información, como propone la periodista Gina Masullo Chen en un texto publicado en Nieman Lab. Según Chen (2012: web), "La mezcla de medios me ofreció - acababa de mudarse de un país de huracanes tres semanas antes de la tormenta una experiencia multimedia, que yo llamaría, incomparable con las viejas épocas de medios impresos, televisión y radio". Eso ocurrió debido a la gran cantidad de informaciones existente en los medios sociales, muchos de ellos con la documentación audiovisual, un hecho que permitió realizar una comparación entre las diversas noticias sobre el tema y la publicación segura por parte de los medios tradicionales. Sin embargo, fue una superación posible para algunos periodistas, especialmente los que estaban acostumbrados a esa nueva realidad mediática y social, donde la noticia circula entre las personas y los periodistas asumen el papel de descubrirla y comprenderla.

En otro momento de 2012, también en los Estados Unidos, el periódico The New York Times creó una página web distinta para el cubrimiento de la destrucción provocada por otro huracán - Sandy -, que ha dejado Nueva York y Nueva Jersey sin accesibilidad, además de la falta de energía y de comunicación convencional. Para superar la ausencia de noticia por problemas incluso de accesibilidad, el periódico construyó un mapa interactivo con los contenidos publicados por los ciudadanos a partir de sus dispositivos móviles y de la tecnología Foursquare para descubrir si la localización y el registro eran realmente dónde y cuándo decían los usuarios. De esa manera, se configuró un mapa de Manhattan y de la región, con los puntos clave y las imágenes ofrecidas por los ciudadanos. Fue otro paso adelante en el proceso de construcción de la noticia a partir de medios sociales y de la participación ciudadana. 
La página, bautizada como A Map of Readers'Photos of Hurricane Sandy ${ }^{4}$, fue publicada como parte de otras informaciones complementarias, como mapas interactivos sobre la condición climática de la región, la trayectoria del huracán Sandy por los Estados Unidos, etc. Sin embargo, la publicación con mejor audiencia fue la de fotos, que ofreció un registro visual por parte de los ciudadanos, ampliando la circulación del contenido de manera viral.

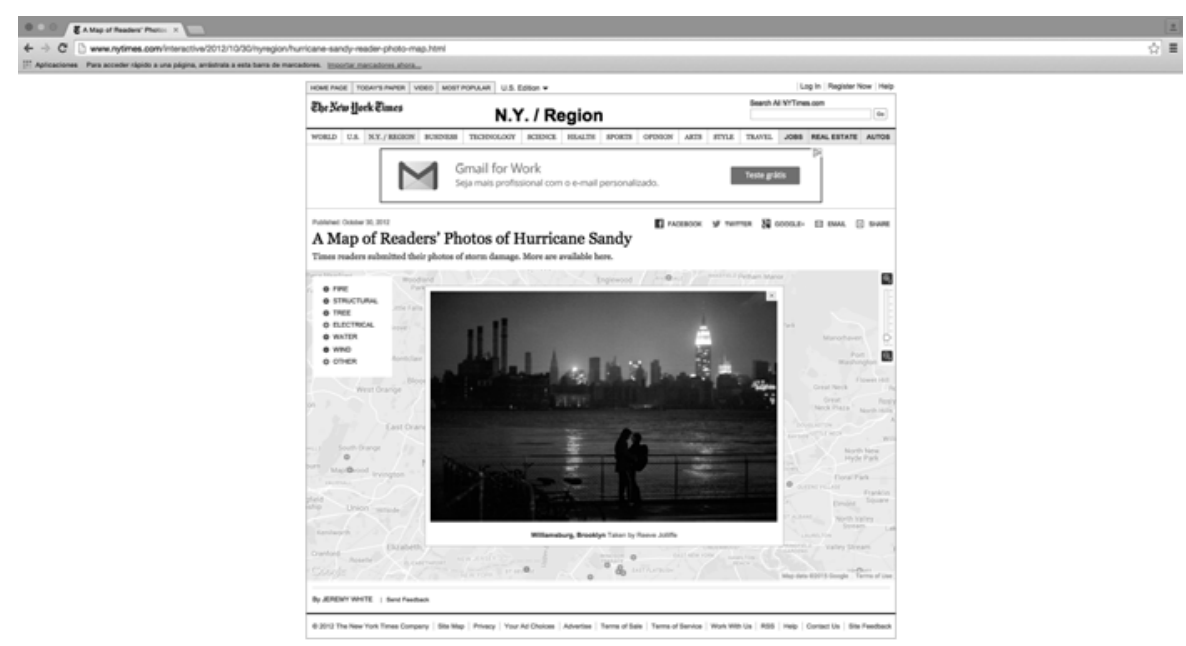

Imagen 1: Manhattan al fondo parcialmente sin luz (publicado por Reeve Jolliffe)

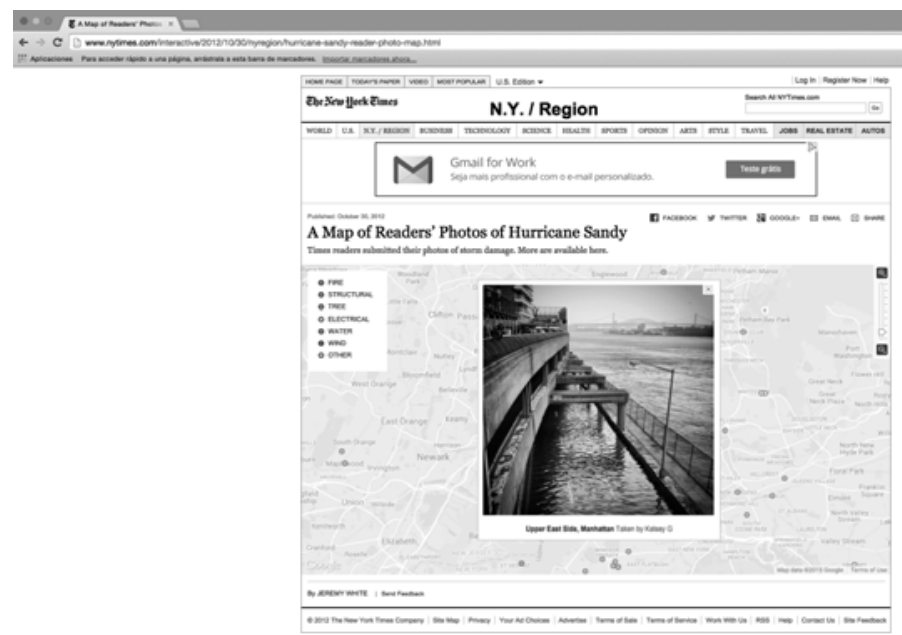

Imagen 2: El agua sobre la calle, en Manhattan (publicado por Kelsey G.)

${ }^{4}$ La página fue creada en conjunto con tecnología Foursquare. Disponible en: http://www.nytimes.com/interactive/2012/10/30/nyregion/hurricane-sandy-reader-photomap.html [Consulta: 19/02/2015]. 
Sin embargo, esos cambios fueron posibles gracias a una redacción mixta, compuesta por profesionales del periodismo y de la tecnología de la información. Solamente así las ideas surgieron y ganaron cuerpo, tornándose reales y posibles. Eso es una prueba de que es fundamental compartir espacios, especialmente hoy, cuando la ecología mediática gana nuevos formatos y pasa a ser un complejo y amplio mundo diverso.

\section{Conclusiones}

El periodismo vive, hoy, un cambio expresivo, que no ocurre solamente fuera de las redacciones, sino también en los espacios profesionales, donde hace tiempo el dominio es del profesional graduado. Además, esto no es se da solamente en Brasil, donde el diploma de periodista no es una exigencia legal desde los primeros años del siglo actual, sino también en los países donde la exigencia jamás ha existido, como por ejemplo en Estados Unidos. Ha cambiada la manera de construir el discurso periodístico, pero también han cambiado el lenguaje, los procesos, la relación con las fuentes (ahora en medios sociales) y la configuración de la redacción.

Pero, estos cambios deben acoplarse a una nueva manera de pensar por parte de los profesionales actuales, especialmente en lo que a la relación con las fuente se refiere (ahora por detrás de una cuenta en Twitter o cosa parecida) y el trabajo compartido con profesionales de otras profesiones, como los del ámbito de la tecnología de la información. De hecho, los profesionales de tecnología de la información deben cambiar la manera de trabajar y pasar a aceptar las distintas miradas que contemplan al usuario y la accesibilidad, aunque eso ofrezca una dificultad de programación.

Sin embargo, estos cambios son inevitables y naturales. El ciudadano contemporáneo "nace" con esas habilidades - la digital y la narrativa -, lo que obliga a que la actividad periodística se ajuste. El creciente desarrollo de aplicaciones que proporcionan la programación de herramientas y páginas web, así como la producción de contenidos hipermediáticos, posibilita el desarrollo de espacios digitales de carácter informativo por parte de los ciudadanos. Y más: con posibilidades narrativas que se aproximan al videojuego y al entretenimiento acompañado de información, o "infoentretenimiento" (Américo et al., 2014: 126), estos contenidos pasan a competir de manera desigual con las tradicionales plataformas informativas.

Pero hay un reto a superar: la noticia frente al Big Data y los medios sociales. Aunque parezca sin credibilidad, estos espacios colectivos ofrecen informaciones muchas veces parecidas a lo que se puede conseguir en la calle. Otras veces, lo que se obtiene a partir de los medios sociales puede ser superior a lo que se consigue solamente hablando con las fuentes. Solo es necesario que los periodistas acepten esa nueva realidad para el desarrollo de la profesión.

En definitiva, consideramos que estos cambios son inevitables y deberían darse directamente en las clases de las universidades, no solamente con la implantación de asignaturas adaptadas a los programas de periodismo basadas en discusiones sobre tecnología, sino también a partir de una formación de docentes, donde las asignaturas tuviesen como responsables profesionales de tecnología, así como a través del establecimiento de actividades interdisciplinarias con otras asignaturas estrictamente periodísticas que necesiten de un upgrade experimental en el campo binario. Se trata de 
una inevitable transformación del periodismo, y no es solamente una impresión apocalíptica del final o la pérdida de control, como apunta Costa (2014), entre otros académicos más conservadores.

\section{Referencias bibliográficas}

AMERICO, Marcos; VIEIRA, Eloiza; y SOTTOVIA, Angelo (2014): "Realidade interativa: protótipo de telejornal interativo para TV Digital baseado no conceito de infotretenimento". Revista Trampas de la Comunicación y la cultura. 77, vol. IV, en: http://www.revistatrampas.com.ar/2014/10/realidade-interativa-prototipode.html [Consulta: 23 de enero de 2015].

CHEN, Gina Masullo (2012): "Hurricane Isaac coverage shows the promise of hybrid model for news". Nieman Lab, 31/08/2012, en: http://www.niemanlab.org/2012/08 /hurricane-isaac-coverage-shows-the-promise-of-a-hybrid-model-for-news [Consulta: 10 de enero de 2015].

COSTA, Luciano M. (2014): “O novo sistema do poder". Observatório da Imprensa, 827, 02/12/2014, en: http://www.observatoriodaimprensa.com.br/news/view /o_novo_sistema_de_poder [Consulta: 13 de enero de 2015].

DADER, José Luis (1997): Periodismo de precisión. Vía socioinformática de descubrir noticias. Madrid, Síntesis.

FLORES, Jesús (2014): Ciberperiodismo: nuevos medios, perfiles y modelos de negocio en la red. Lima, Fondo Editorial USMP.

LAGE, Nilson (2003): A reportagem. Rio de Janeiro, Record.

LEVINSON, Paul (2012): New new media. New York, Pinguim.

MANOVICH, Lev (2013): Software takes command. New York/London, Bloomsbury.

RENÓ, Denis (2014): “Transmedia Journalism and the New Media Ecology: possible languages". En: RENÓ, Denis; CAMPALANS, Carolina; GOSCIOLA, Vicente; y RUIZ, Sandra: Periodismo transmedia: entre teorías y prácticas. Barcelona, Oberta Press.

RENÓ, Luciana y FLORES, Jesús (2014): "Periodismo de datos en el mundo transmedia”. En: RENÓ, Denis; CAMPALANS, Carolina; GOSCIOLA, Vicente; y RUIZ, Sandra: Periodismo transmedia: entre teorías y prácticas. Barcelona, Oberta Press.

THOMPSON, John (1998): A mídia e a modernidade: uma história social da mídia. Petrópolis, Vozes. 